\title{
Tree-level new physics searches in $B$ decays to $\tau$ leptons at Belle
}

\section{Philipp Hamer*}

II. Institute of Physics, Georg-August University of Göttingen

E-mail: philipp.hamer@phys.uni-goettingen.de

We present three analyses of $B$ meson decays to $\tau$ leptons at the Belle experiment, using the full Belle data sample of $772 \times 10^{6} B \bar{B}$ pairs collected at the $\Upsilon(4 S)$ resonance at the KEKB asymmetric energy $e^{+} e^{-}$collider. A first search for the decay $B^{0} \rightarrow \pi^{-} \tau^{+} v_{\tau}$ using hadronic tag is presented, reaching a significance of $2.4 \sigma$. An upper limit of $\mathscr{B}\left(B^{0} \rightarrow \pi^{-} \tau^{+} v_{\tau}\right)<2.5 \times 10^{-4}$ is obtained at the $90 \%$ confidence level. The measurement of $R\left(D^{(*)}\right)=\mathscr{B}\left(B \rightarrow D^{(*)} \tau \nu\right) / \mathscr{B}\left(B \rightarrow D^{(*)} \ell v\right) \quad(\ell=e, \mu)$, is discussed next. The $\tau$ lepton is reconstructed in its leptonic decays. We obtain a result of $R(D)=0.375 \pm 0.064 \pm 0.026$ and $R\left(D^{*}\right)=0.293 \pm 0.038 \pm 0.015$. The results are consistent with previous measurements and do not show a significant deviation from the predictions of the Standard Model. The third analysis presented here is the measurement of the leptonic decay $B^{+} \rightarrow \tau^{+} v_{\tau}$ is presented, where a semileptonic tag is used. We obtain $\mathscr{B}\left(B^{+} \rightarrow \tau^{+} v_{\tau}\right)=(1.25 \pm 0.28 \pm 0.27) \times 10^{-4}$, which is in good agreement with the Standard Model calculations.

The European Physical Society Conference on High Energy Physics 22-29 July 2015

Vienna, Austria

${ }^{*}$ Speaker. 


\section{Introduction}

We present three analyses of (semi-)leptonic $B$ meson decays to a $\tau$ lepton, performed on the full Belle data sample containing $772 \times 10^{6} B \bar{B}$ pairs, collected at the $\Upsilon(4 S)$ resonance at the asymmetric $e^{+} e^{-}$collider KEKB [1]. New particles, like a charged Higgs, may contribute to these decays at the tree-level which could lead to observable deviations between experimental result and Standard Model (SM) calculations. First, we present the search for $B^{0} \rightarrow \pi^{-} \tau^{+} v_{\tau}{ }^{1}$. Next, we discuss the measurement of $R\left(D^{(*)}\right.$ by Belle as well as the combination of the results by BaBar, Belle and LHCb. Third, the latest Belle analysis of the decay $B^{+} \rightarrow \tau^{+} v_{\tau}$ using semileptonic tag is presented.

\section{Belle experiment}

The Belle detector is a large-solid-angle magnetic spectrometer that consists of a silicon vertex detector (SVD), a 50-layer central drift chamber (CDC), an array of aerogel threshold Cherenkov counters (ACC), a barrel-like arrangement of time-of-flight scintillation counters (TOF), and an electromagnetic calorimeter comprised of $\mathrm{CsI}(\mathrm{Tl})$ crystals (ECL) located inside a super-conducting solenoid coil that provides a $1.5 \mathrm{~T}$ magnetic field. An iron flux-return located outside of the coil is instrumented to detect $K_{L}^{0}$ mesons and to identify muons (KLM). The detector is described in detail elsewhere [2].

\section{Search for $\mathbf{B}^{0} \rightarrow \pi^{-} \tau^{+} \nu_{\tau}$}

We present the first search for $B^{0} \rightarrow \pi^{-} \tau^{+} \nu_{\tau}$ [3] in this section. The theoretical description of the decay at the tree-level involves the leptonic and hadronic current. The hadronic current is given by [4]

$$
\left\langle\pi^{-}\left|u \gamma_{\mu} \bar{b}\right| B^{0}\right\rangle=f^{+}\left(q^{2}\right)\left[2 p_{\mu}+\left(\frac{1-m_{B}^{2}-m_{\pi}^{2}}{q^{2}}\right) q_{\mu}\right]+f^{0}\left(q^{2}\right) \frac{m_{B}^{2}-m_{\pi}^{2}}{q^{2}} q_{\mu}
$$

where $f^{+}$and $f^{0}$ are the vector and scalar $B \rightarrow \pi$ form factors, respectively. The ratio $R(\pi)=$ $\mathscr{B}(B \rightarrow \pi \tau v) / \mathscr{B}(B \rightarrow \pi \ell v)$ with $\ell=e, \mu$ depends only on the ratio of the two form factors $f^{0} / f^{+}$ in the SM [4]. Using lastest lattice QCD calculations for the two form factors [5], one obtains $\mathscr{B}\left(B^{0} \rightarrow \pi^{-} \tau^{+} v_{\tau}\right)=(9.35 \pm 0.38) \times 10^{-5}[6]$. The goal of the analysis is to find evidence for the decay, which would allow to measure $R(\pi)$.

The decay $B^{0} \rightarrow \pi^{-} \tau^{+} v_{\tau}$ contains, depending on the decay channel of the $\tau$ lepton, 2-3 neutrinos, which can not be detected. In order to identify possible signal candidates, we try to reconstruct one of the $B$ mesons, the $B_{\text {tag }}$, fully in its hadronic decay. The full hadronic reconstruction at Belle is performed using an algorithm on NeuroBayes [7], which reconstructs $B^{0}$ and $B^{+}$mesons in a total of 1104 different hadronic decay final states.

We select events events with a reconstructed $B^{0}$ meson and search for the signal decay in the remainder of the event. The $\tau$ lepton is reconstructed in the four decay channels $\tau^{+} \rightarrow e^{+} v_{e} \bar{v}_{\tau}$, $\tau^{+} \rightarrow \mu^{+} v_{\mu} \bar{v}_{\tau}, \tau^{+} \rightarrow \pi^{+} \bar{v}_{\tau}$, and $\tau^{+} \rightarrow \rho^{+} \bar{v}_{\tau}$. For charged tracks that are neither identified as

\footnotetext{
${ }^{1}$ Throughout this paper, the inclusion of the charge-conjugate mode process is implied unless otherwise stated.
} 
electron nor muon, a kaon veto is applied and all passing tracks are identified as pions. A signal candidate therefore contains exactly two oppositely charged tracks, of which at least one is identified as a $\pi^{ \pm}$. Muons are required to have a momentum of at least $800 \mathrm{MeV} / \mathrm{c}$ in order to be correctly identified. Many muons from the signal decay are slower than this requirement and are then reconstructed in the $\tau \rightarrow \pi^{2}$ channel. We reconstruct a $\rho$ meson in events with two $\pi^{ \pm}$by combining them with the $\pi^{0}$ candidates and require $M_{\pi^{+}} \pi^{0} \in[625 ; 925] \mathrm{MeV} / \mathrm{c}^{2}$. Due to the broad width of the $\rho$ meson, the reconstruction is not always successful, in which case, the event is reconstructed in the $\tau \rightarrow \pi$ channel. Events containing a $K_{L}$ without energy deposition to the ECL are vetoed. We further separate signal and background events using boosted decision trees (BDT), where for each reconstruction channel, one BDT classifier is trained. An important event variable is the extra energy in the ECL, $E_{\mathrm{ECL}}$. It is defined as all energy deposited in the ECL by particles that are not associated to the $B_{\mathrm{tag}}$, nor the two charged particles from the signal decay. For a signal event, this variable peaks at zero while it assumes higher values for most background events. The semileptonic decays $B^{0} \rightarrow \pi^{-} \ell^{+} v_{\ell}(\ell=e, \mu)$ result in the same final state as the signal decay and also contain no extra energy. The missing mass, given by $M_{\text {miss }}^{2}=\left(p_{\text {beam }}-p_{B_{\text {tag }}}-p_{\pi}-p_{\tau \text {-daughter }}\right)^{2}$, is used to reject these events. As the above mentioned decay only contains one neutrino, $M_{\text {miss }}^{2}$ peaks at zero, while it takes higher values for the signal decays and we require $M_{\text {miss }}^{2}>2.2 \mathrm{GeV}^{2} / \mathrm{c}^{4}$ in the leptonic $\tau$ reconstruction channels.

A fit in $E_{\mathrm{ECL}}$ is performed to obtain the number of signal events and to compute the observed significance level. In the fit, the signal strength and the contribution of the $B^{0} \rightarrow X_{c}$ decays are floating parameters, while the other contributions are fixed to the Monte Carlo (MC) prediction. The systematic uncertainties are included as nuisance parameters in the likelihood description and a toy Monte Carlo approach is used to calculate the significance level. The $\tau \rightarrow \mu$ reconstruction channel does not increase the expected significance and is not used in the final result. In the simultaneous fit in the three remaining $\tau$ decay modes, we observe $52 \pm 24$ signal events, shown in Fig. 1. We obtain a significance level of $2.4 \sigma$ and compute an upper limit of $\mathscr{B}\left(B^{0} \rightarrow \pi^{-} \tau^{+} v_{\tau}\right)<$ $2.5 \times 10^{-4}$ at the $90 \%$ confidence level.

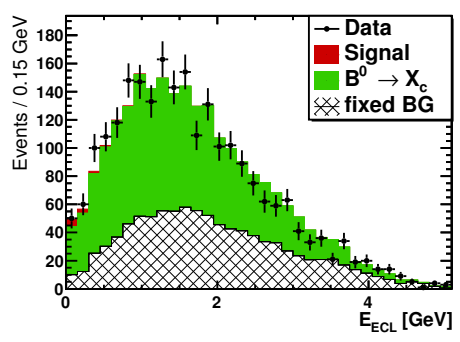

(a)

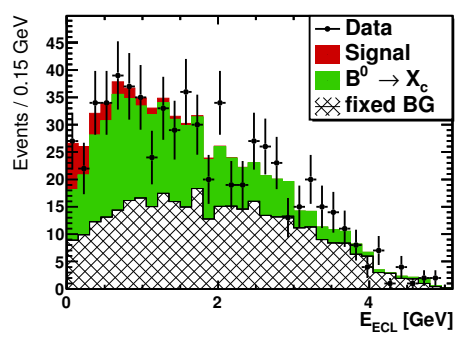

(b)

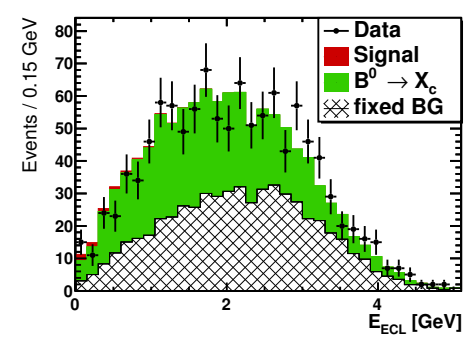

(c)

Figure 1: Simultaneous fit in $E_{\mathrm{ECL}}$ in the three $\tau$ reconstruction modes (a) $\tau \rightarrow e$, (b) $\tau \rightarrow \pi$ and (c) $\tau \rightarrow \rho$.

\footnotetext{
${ }^{2}$ In order to improve readability, the reconstruction channels are written in terms of the charged particle, only.
} 


\section{Measurement of $\mathbf{R}(D)$ and $R\left(D^{*}\right)$}

Similar to above, a charged Higgs boson may contribute to the decay $B \rightarrow D^{(*)} \tau \nu_{\tau}$ and most systematic uncertainties cancel in the ratios $R\left(D^{(*)}\right)=\mathscr{B}\left(\bar{B} \rightarrow D^{(*)} \tau^{-} \bar{v}_{\tau}\right) / \mathscr{B}\left(\bar{B} \rightarrow D^{(*)} \ell^{-} \bar{v}_{\ell}\right)$, $(\ell=e, \mu)$. Previous measurements by BaBar [8] show a deviation of $2.4 \sigma$ from the SM prediction, though the 2HDM type II is not able to describe their result.

The Belle analysis presented here [9] uses the full Belle data sample. We try to fully reconstruct one $B$ meson in its hadronic decay. On the signal side, we reconstruct the $D^{+}$meson in the decays to $K^{-} \pi^{+} \pi^{0}, K_{S}^{0} \pi^{+}, K_{S}^{0} \pi^{+} \pi^{0}$, and $K_{S}^{0} \pi^{+} \pi^{+} \pi^{-}$; the $D^{0}$ meson to $K^{-} \pi^{+}, K^{-} \pi^{+} \pi^{+} \pi^{-}$, $K^{-} \pi^{+} \pi^{0}, K_{S}^{0} \pi^{0}$, and $K_{S}^{0} \pi^{+} \pi^{-}$; the $D^{*+}$ meson to $D^{0} \pi^{+}$and $D^{+} \pi^{0}$; and the $D^{* 0}$ meson to $D^{0} \pi^{0}$ and $D^{0} \gamma$. We reconstruct the $\tau$ lepton in its leptonic decays, $\tau^{-} \rightarrow \ell^{-} \bar{v}_{\ell} v_{\tau}(\ell=e, \mu)$ and obtain four different data samples with the final states $D^{0} \ell, D^{+} \ell, D^{* 0} \ell$, and $D^{*+} \ell$. No further tracks or $\pi^{0}$ are allowed in the remainder of the event, and the charge of the reconstructed particles has to sum to zero. The missing mass squared is given by $M_{\text {miss }}^{2}=\left(p_{\text {beam }}-p_{B_{\text {tag }}}-p_{D^{(*)}}-p_{\ell}\right)^{2}$, and the momentum transfer to the lepton pair $q^{2}=\left(p_{B}-p_{D^{(*)}}\right)^{2}$. Events are rejected unless $M_{\text {miss }}^{2} \in[-0.2 ; 8.0] \mathrm{GeV}^{2} / \mathrm{c}^{4}$ and $q^{2}>4 \mathrm{GeV}^{2} / \mathrm{c}^{2}$. Events containing only one neutrino dominate the low $M_{\text {miss }}^{2}$ region around zero, while the variable takes higher values in events with a $\bar{B} \rightarrow D^{(*)} \tau^{-} \bar{v}_{\tau}$ decay. The reconstruction sample is divided at $M_{\text {miss }}^{2}=0.85 \mathrm{GeV}^{2} / \mathrm{c}^{4}$, and the $\bar{B} \rightarrow D^{(*)} \ell^{-} \bar{v}_{\ell}$ yield obtained from a fit in $M_{\text {miss }}^{2}$. The PDFs for the fit are provided by smoothed histograms, created from the MC prediction. In the signal enhanced region $M_{\text {miss }}^{2}>0.85 \mathrm{GeV}^{2} / \mathrm{c}^{4}$, a neural network is trained for each of the four $\tau$ signal samples, in order to separate the signal decays from the main $D^{* *}$ background decays. The most important input variable is the extra energy in the ECL, $E_{\mathrm{ECL}}$, defined as all energy in the ECL deposited by particles not associated with $B_{\mathrm{tag}}$ nor the $D^{(*)} \ell$ signal side reconstruction. The neural network output $o_{\mathrm{NB}}$ is transformed into

$$
o_{\mathrm{NB}}^{\prime} \equiv \log \frac{o_{\mathrm{NB}}-o_{\min }}{o_{\max }-o_{\mathrm{NB}}},
$$

where $o_{\min }$ and $o_{\max }$ are the minimum and maximum network output variables, respectively, in the selected data sample. The transformed network output has a smooth shape and can be described well with bifurcated Gaussian functions. A simultaneous fit in all four data samples is performed. The $o_{\mathrm{NB}}^{\prime}$ distribution and the fit is shown in Fig. 2.

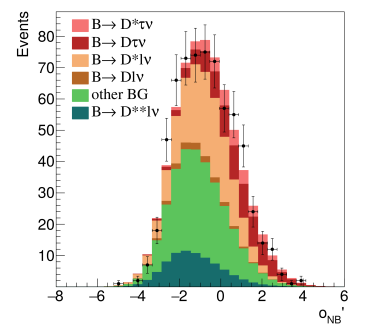

(a)

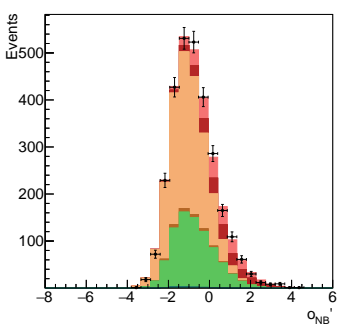

(b)

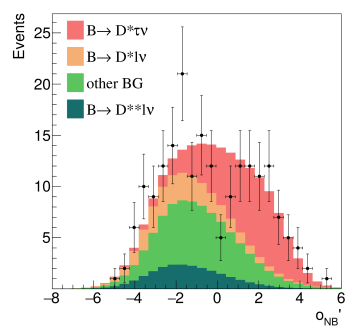

(c)

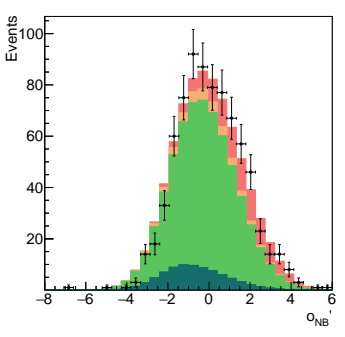

(d)

Figure 2: Distribution and best-fit result in $o_{\mathrm{NB}}^{\prime}$ in the four data samples (a) $D^{+} \ell$, (b) $D^{0} \ell$, (c) $D^{*+} \ell$, and (d) $D^{* 0} \ell$. 
Including all systematic uncertainties, we obtain

$$
\begin{aligned}
R(D) & =0.375 \pm 0.064 \pm 0.026 \\
R\left(D^{*}\right) & =0.293 \pm 0.038 \pm 0.015 .
\end{aligned}
$$

The result lies between the SM prediciton and the $\mathrm{BaBar}$ measurement and is compatible with both. It is also compatible with the 2HDM type II model in the region around $\tan \beta / m_{H^{+}}=0.5 \mathrm{c}^{2} / \mathrm{GeV}$, as shown in Fig. 3a. The Heavy Flavor Averaging Group (HFAG) prepared a combination of the results from BaBar, Belle and $\mathrm{LHCb}$ for this conference, shown in Fig. 3b. The combined result in the $R(D)-R\left(D^{*}\right)$ plane shows a deviation of $3.9 \sigma$ from the SM prediction.

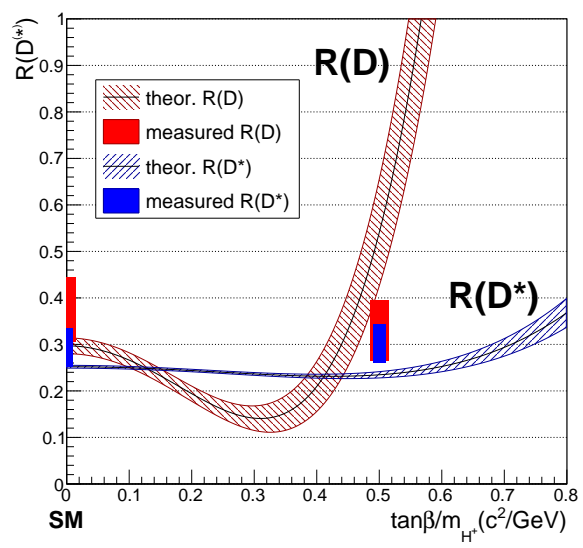

(a)

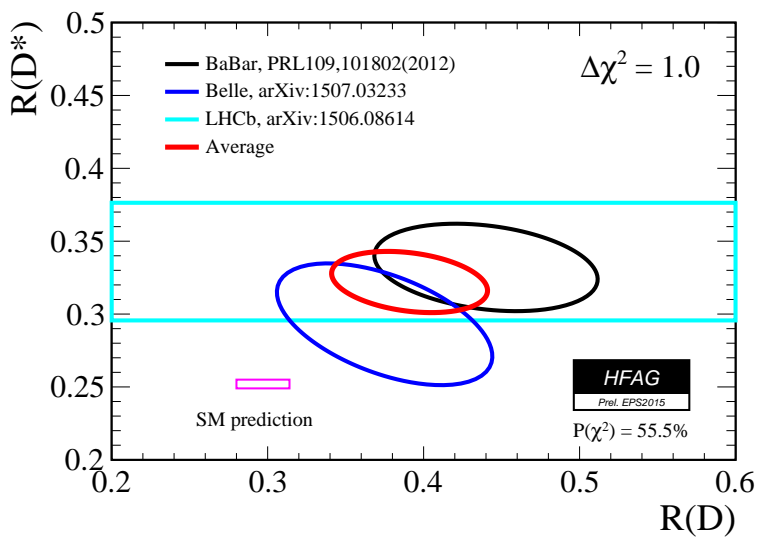

(b)

Figure 3: (a) Result of this measurement and comparision with the 2HDM type II. (b) Results of recent $R\left(D^{(*)}\right)$ measurements by BaBar, Belle, and $\mathrm{LHCb}$ and their deviation from the SM prediction.

\section{Measurement of $\mathscr{B}\left(\mathbf{B}^{+} \rightarrow \tau^{+} v_{\tau}\right)$}

The leptonic $B^{+}$decay into a $\tau v_{\tau}$ pair may be mediated by new, heavy particles. The additional decay diagram can alter the branching ratio with regard to the SM prediction. Previous measurements of $\mathscr{B}\left(B^{+} \rightarrow \tau^{+} v_{\tau}\right)[12,13,14]$ are all slightly above the SM prediction, except for the Belle measurement with hadronic tag [11], which is in very good agreement with it. Here, we present the latest measurement of $\mathscr{B}\left(B^{+} \rightarrow \tau^{+} v_{\tau}\right)$ by Belle [10], which is complementary to the previous Belle measurement using hadronic tag. Instead of using a full hadronic reconstruction of one of the $B$ mesons, we use a semileptonic tag in this analysis. One of the $B$ mesons is reconstructed in $B^{+} \rightarrow \bar{D}^{(*) 0} \ell^{+} v_{\ell}(\ell=e, \mu)$, using a neural network based on NeuroBayes. While the semileptonic tag results in one additional neutrino in the event, the reconstrution efficiencies is higher than for the hadronic tag. The $\tau$ lepton is reconstructed in the decays $\tau^{+} \rightarrow e^{+} v_{e} \bar{v}_{\tau}, \tau^{+} \rightarrow \mu^{+} v_{\mu} \bar{v}_{\tau}$, $\tau^{+} \rightarrow \pi^{+} \bar{v}_{\tau}$, and $\tau^{+} \rightarrow \rho^{+} \bar{v}_{\tau}$. No further charged track and $\pi^{0}$ is allowed in the event, and the overall charge must sum to zero. Events that contain a $K_{L}$ without energy deposition in the ECL are vetoed. The separation between signal and background events is done by a neural network. 
As in the other analyses presented here, the extra energy in the ECL, $E_{\mathrm{ECL}}$, is an important event variable. We obtain the signal yield from a two dimensional fit in $E_{\mathrm{ECL}}$ and the center-of-mass momentum of the visible $\tau$ daughter particle, $p_{\text {sig }}^{*}$. The contribution from $e^{+} e^{-} \rightarrow q \bar{q}$ events is scaled using off-resonance data, taken at a center-of-mass energy $60 \mathrm{MeV}$ below the $\Upsilon(4 S)$ mass.

We obtain a result of $\mathscr{B}\left(B^{+} \rightarrow \tau^{+} v_{\tau}\right)=(1.25 \pm 0.28 \pm 0.27) \times 10^{-4}$ with a significance of $3.8 \sigma$. The result is consistent with the previous measurement using the semileptonic tagging method [14]. Combining the result presented here, with the result obtained using the hadronic tag [11], taking into account all correlated systematic uncertainties, results in a branching ratio of $\mathscr{B}\left(B^{+} \rightarrow \tau^{+} v_{\tau}\right)=(0.91 \pm 0.19 \pm 0.11) \times 10^{-4}$ with a combined significance of $4.6 \sigma$, which is in good agreement with the SM expectation based on $V_{u b}$ constrains from independent measurements. The previous measurements, as well as this results, are shown in Fig. 4.

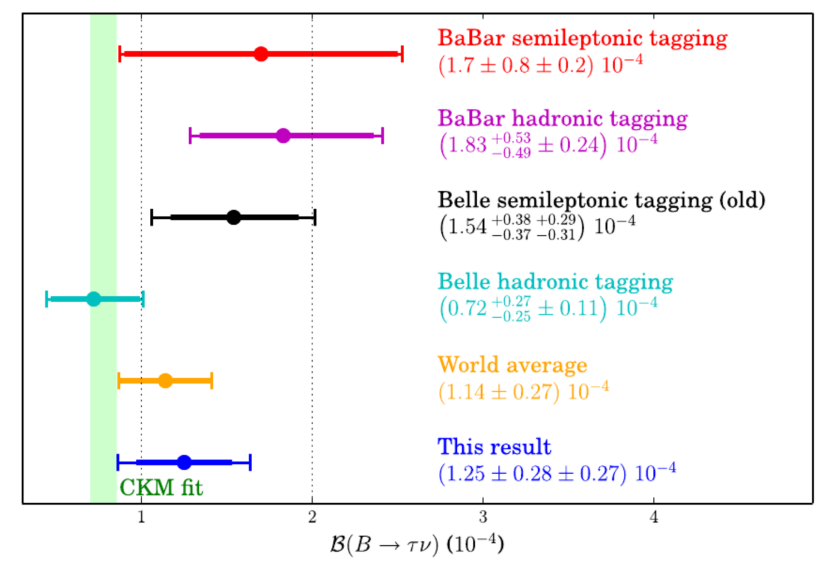

Figure 4: Measurements of $\mathscr{B}\left(B^{+} \rightarrow \tau^{+} v_{\tau}\right)$ and an estimate from the CKMfitter Group.

\section{References}

[1] S. Kurokawa and E. Kikutani, Overview of the KEKB accelerators, Nucl. Instr. and Meth. A 4991 (2003); T. Abe et al., Prog. Theor. Exp. Phys. 2013 03A001 (2013) and references therein.

[2] A. Abashian et al. (Belle Collaboration), Nucl. Instr. and Meth. A 479117 (2002); also see detector section in J. Brodzicka et al., Prog. Theor. Exp. Phys. 2012 04D001 (2012).

[3] P. Hamer et al. (Belle Collaboration), Search for $B^{0} \rightarrow \pi^{-} \tau^{+} v_{\tau}$ with hadronic tagging at Belle, 1509.06521 [hep-ex]

[4] A. Khodjamirian, T. Mannel, N. Offen, and Y.-M. Wang, $B \rightarrow \pi \ell v_{l}$ Width and $\left|V_{u b}\right|$ from $Q C D$ Light-Cone Sum Rules, Phys. Rev. D 83094031 (2011), [1103. 2655 [hep-ph ] ]

[5] J.A. Bailey et al. (Fermilab Lattice, MILC), $\left|V_{u b}\right|$ from $B \rightarrow \pi \ell v$ decays and (2+1)-flavor lattice, Phys. Rev. D 92014024 (2015), [1503.07839 [hep-lat] ]

[6] R. van de Water, D. Du, Private Communication

[7] M. Feindt, et al., A hierarchical NeuroBayes-based algorithm for full reconstruction of B mesons at B factories, Nucl. Instr. and Meth. A 654432 (2011), [1102 . 3876 [hep-ex] ]

[8] J. P. Lees et al. (BaBar Collaboration), Evidence for an excess of $\bar{B} \rightarrow D^{(*)} \tau^{-} \bar{v}_{\tau}$ decays, Phys. Rev. Lett. 109101802 (2012), [1205. 5442 [hep-ex] ] 
[9] M. Huschle et al. (Belle Collaboration), Measurement of the branching ratio of $\bar{B} \rightarrow D^{(*)} \tau^{-} \bar{v}_{\tau}$ relative to $\bar{B} \rightarrow D^{(*)} \ell^{-} \bar{v}_{\ell}$ decays with hadronic tagging at Belle, 1507.03233 [hep-ex]

[10] B. Kronenbitter et al. (Belle Collaboration), Measurement of the branching fraction of $B^{+} \rightarrow \operatorname{tau}^{+} v_{\tau}$ decays with the semileptonic tagging method, Phys. Rev. D 92051102 (2015),

[1503.05613 [hep-ex]]

[11] K. Hara et al. (Belle Collaboration), Evidence for $B^{-} \rightarrow \tau^{-} \bar{v}_{\tau}$ with a Hadronic Tagging Method Using the Full Data Sample of Belle, Phys. Rev. Lett. 110131801 (2013), [1208.4678 [hep-ex]]

[12] B. Aubert et al. (BaBar Collaboration), A Search for $B^{+} \rightarrow \ell^{+} v_{\ell}$ Recoiling Against $B^{-} \rightarrow D^{0} \ell^{-} \bar{v} X$, Phys. Rev. D 81051101 (2010), [0912.2453 [hep-ex]]

[13] J. P. Lees et al. (BaBar Collaboration), Evidence of $B^{+} \rightarrow \tau^{+} v$ decays with hadronic B tags, Phys. Rev. D 88031102 (2013), [1207.0698 [hep-ex] ]

[14] K. Hara et al. (Belle Collaboration), Evidence for $B^{-} \rightarrow \tau^{-} \bar{v}$ with a Semileptonic Tagging Method, Phys. Rev. D 82071101 (2010), [1006.4201 [hep-ex] ] 